\title{
Cometary Charge Exchange Diagnostics in UV and X-ray
}

\author{
D. Bodewits ${ }^{1, \star}$, D.J. Christian ${ }^{2}$, J.A. Carter ${ }^{3}$, K. Dennerl ${ }^{4}$, I. Ewing $^{2}$, R. Hoekstra ${ }^{5}$, S.T Lepri $^{6}$, \\ C.M. Lisse ${ }^{7}$, and S.J. Wolk ${ }^{8}$ \\ 1 Dept. of Astronomy, University of Maryland, College Park, MD 20742-2421, USA \\ 2 Department of Physics and Astronomy, California State University, 18111 Nordhoff Street, Northridge, CA 91330, USA \\ 3 Department of Physics and Astronomy, University of Leicester, Leicester, LE1 1RH, UK \\ 4 Max-Planck-Institut fur extraterrestrische Physik, Giessenbachstrasse, 85748 Garching, Germany \\ 5 KVI Atomic Physics, University of Groningen, Zernikelaan 25, NL-9747 AA Groningen, The Netherlands \\ 6 The University of Michigan, Ann Arbor, MI 481092143, USA \\ 7 Planetary Exploration Group, Space Department, JHU-APL, 11100 Johns Hopkins Rd, Laurel, MD 20723, USA \\ ${ }^{8}$ Harvard-Smithsonian Center for Astrophysics, 60 Garden Street, Cambridge, MA 02138, USA
}

Received 14 Feb 2012, accepted 14 Feb 2012

Published online later

Key words comets: general - solar wind - atomic processes - radiation mechanism: non-thermal

Since the initial discovery of cometary charge exchange emission, more than 20 comets have been observed with a variety of X-ray and UV observatories. This observational sample offers a broad variety of comets, solar wind environments and observational conditions. It clearly demonstrates that solar wind charge exchange emission provides a wealth of diagnostics, which are visible as spatial, temporal, and spectral emission features. We review the possibilities and limitations of each of those in this contribution.

(c) 2012 WILEY-VCH Verlag GmbH \& Co. KGaA, Weinheim

\section{Introduction}

X-ray and Extreme Ultraviolet (EUV) emission is usually associated with high temperature environments. The discovery that comets are bright emitters in this spectral regime was therefore a big surprise (Lisse et al. 1996; Mumma et al. 1997), and is in strong contrast to our understanding that comets are dirty snowballs surrounded by a gaseous coma with a temperature of approximately $50 \mathrm{~K}$. After the first discovery by ROSAT of the X-ray emission from Comet C/1996 B2 (Hyakutake), a search through the observatory's archives proved that in fact all comets (with total visual magnitude $\mathrm{m}_{v}<12.0$ ) in the inner solar system ( $\leq 2$ AU) had emitted X-rays (Dennerl et al. 1997). The total X-ray power in the $0.2-1.0 \mathrm{keV}$ band was between $0.004-1.2 \mathrm{GW}$, the emission was highly variable in time, and many of the observed comets displayed a characteristic crescent shape.

To explain these surprising observations, numerous possible scenarios were proposed. Amongst them were scattering/fluorescence of solar X-rays (Krasnopolsky 1997), thermal bremsstrahlung associated with collisions of solar wind electrons with cometary neutral gas or dust (Bingham et al. 1997; Northrop et al. 1997; Uchida et al. 1998), electron/proton K- and L-shell ionization (Krasnopolsky 1997), Rayleigh-scattering of solar X-rays by attogram dust particles (Owens et al. 1998;

\footnotetext{
^ Corresponding author: e-mail: dennis@ astro.umd.edu
}

Schulz et al. 2000; Wickramasinghe \& Hoyle 1996), and charge exchange between highly ionized solar wind minor ions and cometary neutral species (Cravens 1997). A comparative study by Krasnopolsky (1997) demonstrated that none of these mechanisms except for the solar wind charge exchange emission and attogram dust scattering could account for the observed luminosities. The discovery of emission lines in the X-ray spectrum of Comet C/1999 S4 (LINEAR) prove that the emission was driven by solar wind charge exchange (Lisse et al. 2001).

Before data from spacecraft monitoring the solar wind became available, observations of cometary ion tails were the only method of probing the solar wind. Even nowadays, comets largely remain the only means to sample regions outside the ecliptic plane. Cometary X-rays in particular have proven to be an excellent tool to study solar wind - neutral gas interactions, because comets have no magnetic field and the wind therefore interacts directly with the neutral gas in the coma. Secondly, the size of the cometary atmosphere (on the order of $10^{5} \mathrm{~km}$ ) allows remote tracking of the ions as they penetrate into the comet's atmosphere, offering a closeup view on the interaction of the two plasmas. Thirdly, since the first observations of cometary X-ray emission, more than 20 comets have been observed with various Xray and UV observatories including ROSAT (Dennerl et al. 1997; Lisse et al. 1996), EUVE (Mumma et al. 1997), BeppoSAX (Owens et al. 1998), Chandra (Bodewits et al. 2007; Christian et al. 2010; Krasnopolsky et al. 2004; 


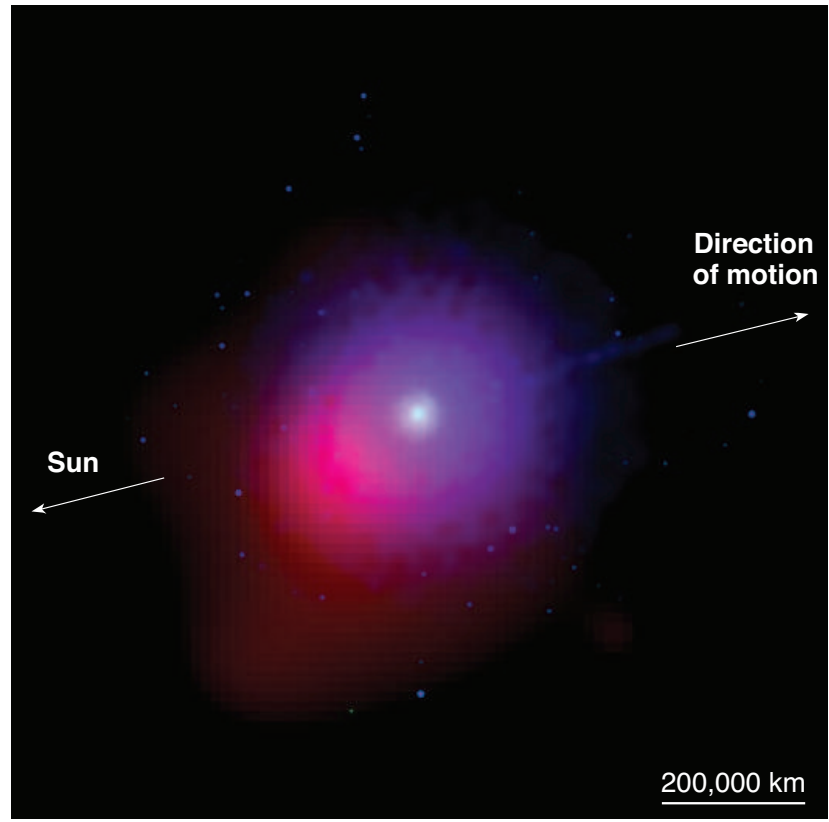

Fig. 1 Swift observations of comet 2007/N3 (Lulin), adapted from Carter et al. (2012). The Swift satellite allows for simultaneous mapping of $\mathrm{OH}$ in the neutral coma (blue) and charge exchange X-ray emission (red). The peak X-ray brightness is offset by about $35,000 \mathrm{~km}$ towards the Sun.

Lisse et al. 2001; Wolk et al. 2009), CHIPS (Sasseen et al. 2006), FUSE (Feldman 2005), XMM-Newton (Dennerl 2012; Schultz et al. 2006), Suzaku (Brown et al. 2010), and Swift (Carter et al. 2012; Willingale et al. 2006). This observational sample offers a broad variety of comets, solar wind environments and observational conditions, clearly demonstrating that cometary charge exchange emission provides a wealth of diagnostics, which are visible as spatial, temporal, and spectral emission manifestations. We will discuss the possibilities and limitations of each of those in this contribution.

\section{Morphology}

The emission morphology is a 3D 'tomography' of the solar wind flow through the neutral gas around the nucleus (Wegmann et al. 2004). In very active comets (gas production rates of $10^{29}$ molecules $\mathrm{s}^{-1}$ or more, Bodewits et al. 2007) observed at phase angles of about 90 degrees, the $\mathrm{X}$-ray emission maps a spherical gas distribution resulting in a characteristic sunward, crescent shape as seen by a remote observer, indicating a coma that is collisionally thick to charge exchange. A good example results from the combined observations of comet 2007/N3 (Lulin), shown in Figure1 (Carter et al. 2012). The Swift observatory is equipped with boresighted UV and X-ray instruments, allowing for

\footnotetext{
1 Unsuccessful attempts to detect X-rays in comets were made using the Einstein satellite, the Rossi X-ray Timing Explorer, and the ASCA observatory (Krasnopolsky et al. 2004).
}

simultaneous mapping of $\mathrm{OH}$ in the neutral coma (blue) and charge exchange X-ray emission (red). The peak X-ray brightness is offset by about $35,000 \mathrm{~km}$ towards the Sun.

Less active comets (gas production rates of $10^{28}$ molecules $\mathrm{s}^{-1}$ or less) have neutral comae that are virtually transparent to the incoming solar wind ions. The X-ray emission typically peaks at the nucleus, and jets or other local enhancements in the coma brighten, as was observed for the jet in 2P/Encke (Lisse et al. 2005) and the curious X-ray morphology surrounding the fragmented comets C/1999 S4 (LINEAR) and 73P/Schwassmann-Wachmann 3 (Lisse et al. 2001; Wolk et al. 2009). Wegmann \& Dennerl (2005) analyzed a cometary x-ray image and deduced the shock location, but this has not yet been fully explored as their technique requires imaging data with a very high signal to noise ratio.

At 1 AU from the Sun, most of the charge exchange reactions take place in the denser parts of the coma within $\approx$ $10^{5} \mathrm{~km}$ from the nucleus (Bodewits et al. 2007). For nearby comets, this often implies that the cometary emission extends well beyond the field of view and requires a careful background subtraction. This introduces an important problem for observers. The diffuse soft X-ray background is variable in time and place, and contains many of the SWCX lines observed in comets resulting from charge exchange in the Earths exosphere and the heliosheath (Carter \& Sembay 2008; Carter et al. 2011; Koutroumpa et al. 2007, 2008). This background subtraction problem was explored in depth for the very extended Comet 17P/Holmes (Christian et al. 2010).

\section{Temporal Variation}

Cometary X-ray luminosity is driven by a combination of the comet's gas production rate, the solar wind flux, and its heavy ion content. Furthermore, for nearby comets the effect of varying distance on the projected effective aperture should be taken into account. The interpretation of cometary X-ray lightcurves is therefore complex, as was first shown for Comet Hyakutake (Neugebauer et al. 2000). Long term Swift XRT and Chandra observations of Comet 9P/Tempel 1 (Lisse et al. 2007; Willingale et al. 2006) demonstrated the richness of this approach (see Fig. 2). Large increases in the X-ray luminosity could be assigned to a large cometary outburst (DOY 2005-180 to 184) followed by the interplanetary manifestation of a Coronal Mass Ejection (DOY 2005-188 to 190). A novel approach has recently been presented in Carter et al. (2012), who directly constrained the cometary gas production rates and related this to the observed X-ray variability by employing Swift's co-aligned instrument suite, simultaneously observing comet C/2007 N3 (Lulin) in UV and X-rays. It is of note that the optical brightness of comets is driven by their dust content, which in itself is only weakly coupled to their overall gas activity (A'Hearn et al. 1995; Jorda et al. 2008). As the solar wind also has no visual manifestation in interplan- 


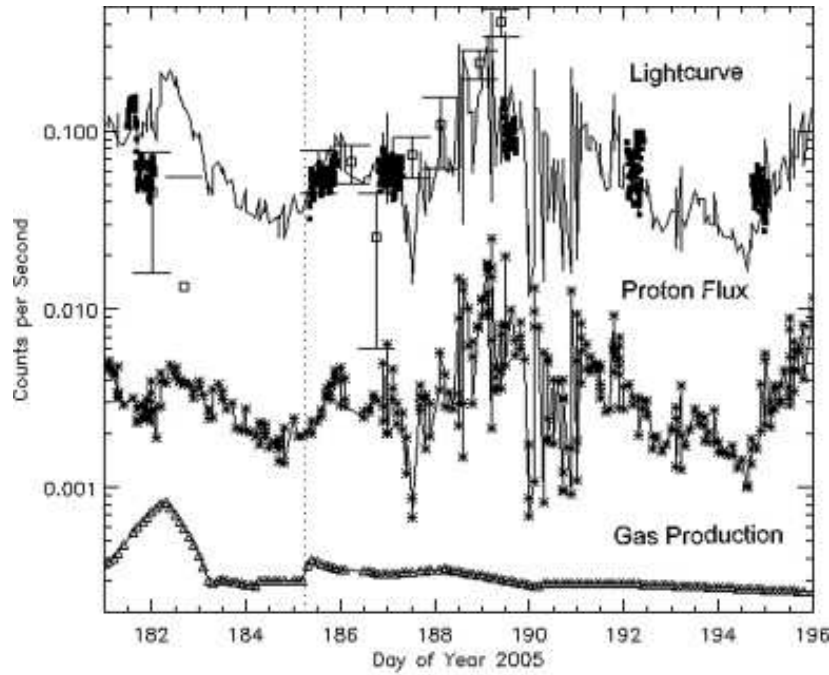

Fig. 2 The X-ray light curve is the result of comet activity and solar wind ion flux. Shown here are Chandra (filled squares) and Swift observations (open squares, Willingale et al. 2006 - normalized to the Chandra measurements) of Comet 9P/Tempel 1 around the time of Deep Impact. For comparison, we also show estimated water (gas) production rates (triangles, bottom), solar wind proton fluxes (stars, middle), and the product of these two, which is the predicted X-ray lightcurve (solid line, top). Figure adapted from Lisse et al. (2007).

etary space, there is therefore no simple relation between the optical and X-ray luminosities of comets.

The solar wind is highly variable in time and its ion composition can change dramatically over the course of less than a day due to variations in the solar source regions and dynamic changes in the solar wind itself. While the solar wind is sampled at the Earth's first Lagrangian Point L1 by several spacecraft, to accurately model SWCX lightcurves solar wind properties have to be extrapolated to the position of the comet. Full MHD propagation models are available but are only applicable to comets in the equatorial plane. These models currently offer an accuracy of a day at best (see e.g. Fry et al. (2003) and references therein), and only consider the wind's bulk properties, not its composition. A first order estimate of the state of the solar wind can be achieved using corotational mapping of the solar wind (Neugebauer et al.2000), but this method only considers the Sun's rotation and the wind's bulk velocity, and performs poorly propagating dynamical structures in the wind. Latitudinal and corotational separations imply large inaccuracies in any solar wind mapping procedure and limit the comparison of solar wind data with X-ray observations to nearby comets (Bodewits et al. 2007; Wolk et al. 2009).

\section{Spectroscopy}

Charge exchange reactions are quasi-resonant processes, and depend strongly on properties of both the neutral
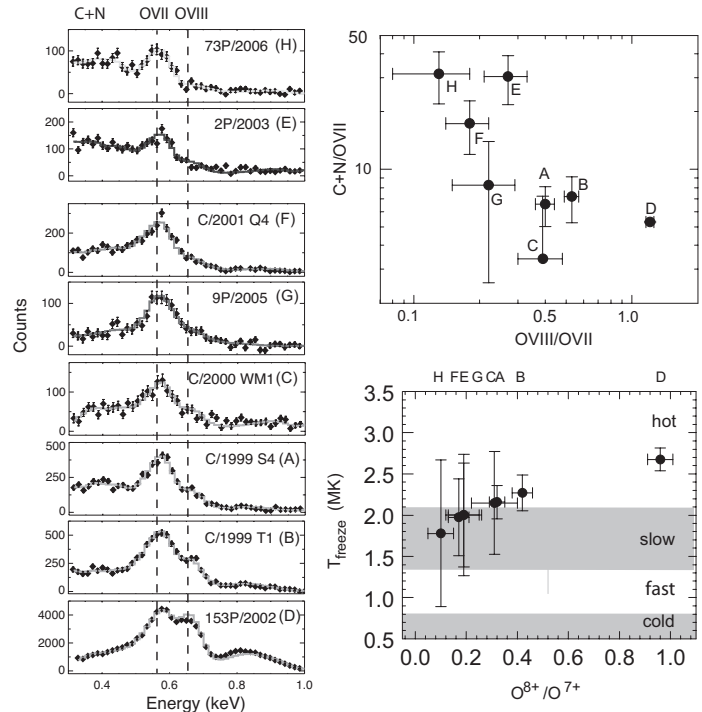

Fig. 3 Comparison of 8 different comets observed by Chandra between 2000 - 2006, adapted from (Bodewits et al. 2007). Left: X-ray spectra, organized by color. Top right: Flux ratios (colors) of the spectra. The low energy feature $(\mathrm{C}+\mathrm{N})$ is anti-correlated to the oxygen flux ratio. Bottom right: Oxygen charge states derived from the Chandra spectra and their corresponding freeze-in temperatures. The shaded areas indicate typical freeze-in temperatures for different solar wind states.

and the ionized gas Cravens (2002). The resulting emission therefore provides a unique window on their interactions. For example, within fusion experiments both Doppler shifts of charge exchange emission lines and their absolute and relative intensities are used to provide information on local plasma parameters such as temperatures, velocities and abundances and charge state of the interacting plasmas (Anderson et al. 2000; Hoekstra et al. 1998; Isler 1994; von Hellermann et al. 1991).

Current charge exchange investigations however are limited by observational constraints and sparse experimental data. Most cometary spectra have been obtained using Chandra's ACIS-S instrument, which provides moderate energy resolution (110 eV FWHM) in the 300 to 2000 eV energy range. Several attempts have been made to extract ionic abundances from the X-ray spectra (Haberli et al. 1997; Kharchenko \& Dalgarno 2000, 2001; Krasnopolskv 2004; Krasnopolsky et al. 2002; Lisse et al. 2001, 2005; Schwadron \& Cravens 2000; Wegmann et al. 2004, 1998). However, the multitude of emission lines from hydrogenand helium like heavy ions in this regime implies that individual SWCX lines are not resolved, often rendering leastsquare fits degenerate. More favorable approaches minimize the degrees of freedom by grouping all emission lines of each ion based on compilations of experimental and theoretical work (Beiersdorfer et al. 2003; Bodewits et al. 2007).

The overall shape of cometary SWCX spectra is predominantly determined by the state of the solar 
wind (Bodewits et al. 2007; Kharchenko \& Dalgarno 2001; Schwadron \& Cravens 2000). During solar minimum the solar wind can be simplified to the stratification of a slow, low-latitude wind originating about the solar equator and a higher-latitude, faster wind originating from polar regions. In this bi-modal state the polar wind has a lower charge state than the wind at lower latitudes. This simple picture is complicated by the presence of transient phenomena (corotating interaction regions and coronal mass ejections), and by the chaotic state of the solar corona around solar maximum (Geiss et al. 1995). In low resolution spectra, these states manifest themselves as 'color differences', i.e. in the relative strength of the low energy $(<500 \mathrm{eV})$, the OVIII emission at $561 \mathrm{eV}$ and the OVIII emission at $653 \mathrm{eV}$. A comparison of 8 comets observed with Chandra showed a clear separation between the spectra that are dominated by the low energy component, and the 'oxygen-dominated' spectra. The spectra dominated by emission $>500 \mathrm{eV}$ show a gradual increase in the oxygen ionic ratio (Fig. 3). Because the solar wind is a collisionless plasma, this trend can be understood in terms of the freeze-in temperature of the solar wind source region (Fig. (4). The slow wind associated with streamers typically has freeze-in temperatures of 1.3 - 2.1 MK (Bryans et al. 2009; Zurbuchen et al. 2002). With lower freeze-in temperatures, such as found in solar wind associated with coronal holes or at higher solar latitudes, the oxygen charge state distribution decreases accordingly, and most oxygen is found in the $\mathrm{O}^{6+}$ state which emits in the Far UV after charge exchange. Because the low energy component of the spectrum contains both $\mathrm{C}^{5+}$ and $\mathrm{C}^{6+}$ which lines cannot be resolved with the current spectral resolution, it is less sensitive to changes in typical solar wind freeze-in temperatures.

Due to its reliance on a good detection of the O VIII feature, the oxygen ion ratio has limited diagnostic use for fast and cool solar winds with freeze-in temperatures of $1 \mathrm{MK}$ or less, such as the wind associated with coronal holes or the cold wind found at higher solar latitudes (Christian et al. 2010). Ion ratios between H- and He- like Carbon would make excellent probes for this temperature regime (Landi et al. 2012) but their lines cannot be resolved at the spectral resolution currently achievable. Extremely hot solar wind states provide another limiting case. The spectrum of Comet 153P/Ikeya-Zhang cannot be well explained by the Bodewits et al. (2007) SWCX model (Fig. 3). It anomalous spectrum was attributed to the interaction with the interplanetary manifestation of a coronal mass ejection (ICME), likely resulting in many additional unresolved SWCX lines of Fe XV-XX. Both issues will be addressed once $\mathrm{X}$-ray calorimeters become available to observers.

\footnotetext{
2 To avoid confusion, solar wind ions are in superscript notation and emitting ions are notated in linear notation.
}

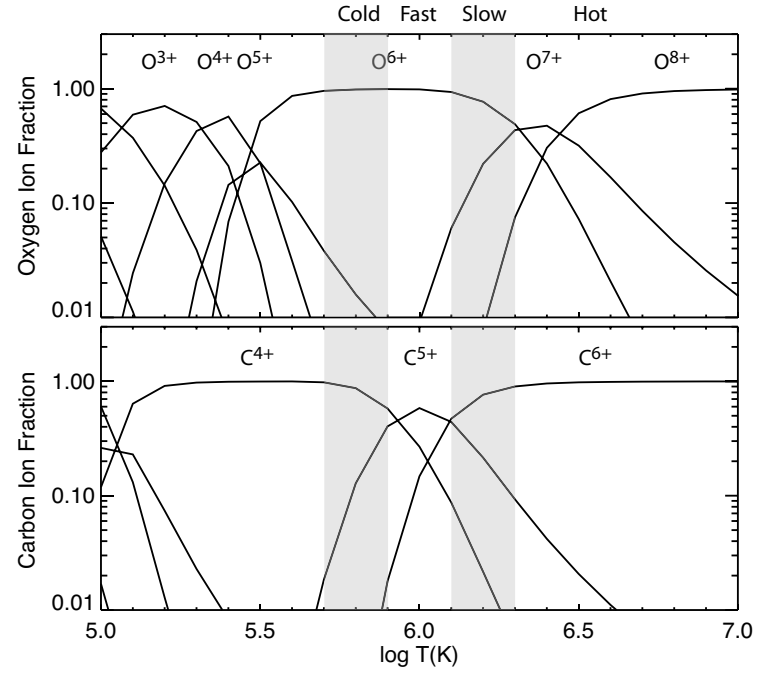

Fig. 4 Relative ion fractions for oxygen (top) and carbon (bottom), as a function of temperature in an optically thin plasma under thermal equilibrium, adapted from Bryans et al. (2009) and Landi et al. (2012). Typical solar wind freeze-in temperatures are indicated by shaded areas.

\subsection{High Energy - Above $1 \mathrm{KeV}$}

SWCX X-ray spectra above $1 \mathrm{keV}$ have not been well studied in comets, although tentative detections of $\mathrm{Mg}$ were presented in the spectrum of the exceptionally Xray bright comet Ikeya-Zhang in Bodewits et al. (2007). Also, Carter et al. (2010) found evidence for Fe XVII - XX, Mg XI - XII, and Si XIII - XIV lines from the Earth's exosphere. Both papers attributed the presence of these highly charged ions due to coronal mass ejection events, which can have freeze-in temperatures of several millions of degrees (Lepri \& Zurbuchen 2004).

Recent work by Ewing et al. (2012) has confirmed the strong 1.3-2.0 keV emission lines from Ikeya-Zhang and detected lines at 1350, 1470, 1840 and $2000 \mathrm{eV}$ that were identified as Mg XI, Mg XII, Si XIII, and Si XIV SWCX lines. They demonstrated that upon close inspection of archival Chandra observations these $\mathrm{Si}$ and $\mathrm{Mg}$ lines were present at a significant level for at least 4 more of the comets.

\subsection{Low Energy - Below $300 \mathrm{eV}$}

The close encounter with comet 73P/Schwassmann-Wachmann 3 (which came within $0.07 \mathrm{AU}$ of Earth in 2006) allowed for (nearly) in situ measurements of the solar wind, enabling a direct test of spectral models. Wolk et al. (2009) compared ion abundances derived from Chandra observations with measurements by the ACE-SWICS instrument. While the derived abundances of $\mathrm{O}^{7+}$ and $\mathrm{C}^{6+}$ were in good agreement with the solar wind data, the obtained relative abundance of $\mathrm{C}^{5+}$ was off by an order of magnitude. Part of this discrepancy might be explained by the lack of experimental charge exchange cross sections or calibra- 

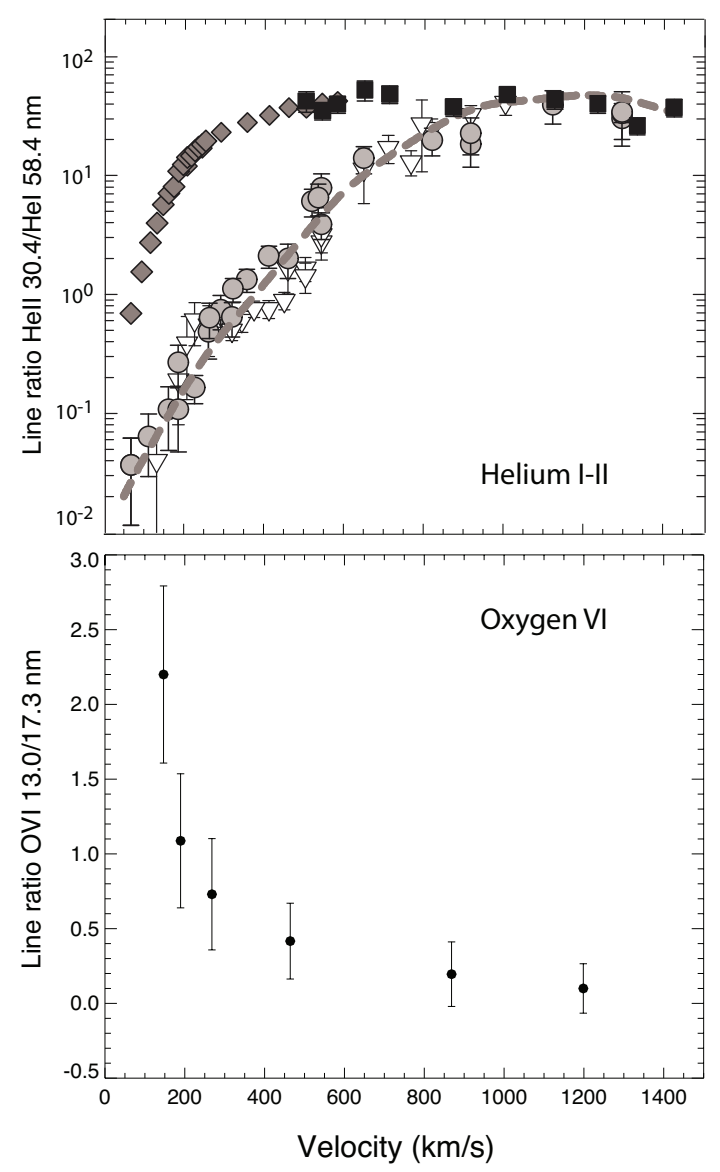

Fig. 5 Charge exchange diagnostics in the Extreme- and Far-UV. Top Panel: Helium line ratios between $30.4 \mathrm{~nm}$ and $58.4 \mathrm{~nm}$ emission following $\mathrm{He}^{2+}$ colliding on $\mathrm{H}_{2}$ (white triangles), $\mathrm{CO}$ (grey circles), and $\mathrm{H}_{2} \mathrm{O}$ (black squares and grey diamonds). Bottom Panel: Velocity dependence of the O VI line ratio between the $1 s^{2} 3 d-1 s^{2} 2 p$ and $1 s^{2} 4 p-$ $1 \mathrm{~s}^{2} 2 \mathrm{~s}$ transitions at 17.3 and $13.0 \mathrm{~nm}$, respectively. Figures adapted from Bodewits \& Hoekstra (2007); Bodewits et al. (2005).

tion issues at the low-energy end of Chandra-ACIS. However, the excess low energy has been a persistent feature. Bodewits et al. (2007) showed that the ratio between $\mathrm{C}^{6+}$ and $\mathrm{C}^{5+}$ ions and the oxygen ion ratio was poorly correlated, ostensibly because $\mathrm{C}^{5+}$ abundances are consistently too large. It is therefore very likely that the source of the discrepancy is related to emission from several ions of species such as $\mathrm{Fe}, \mathrm{Mg}, \mathrm{Si}, \mathrm{Ne}$, and as much of $90 \%$ of the emission around $300 \mathrm{eV}$ should be attributed to species other than $\mathrm{C}^{5+}$ (Brown et al. 2010; Koutroumpa et al. 2008; Sasseen et al. 2006).

\subsection{Ultraviolet}

As was noted above, many SWCX lines fall below the bandwidth covered by current $\mathrm{X}$-ray observatories. The excited states of $\mathrm{H}-$ and $\mathrm{He}-$ like $\mathrm{C}, \mathrm{N}$, and $\mathrm{O}$ decay in a radia- tive cascade which produces photons at X-ray, EUV, and UV wavelengths. In fact, the most abundant ions in the solar wind, $\mathrm{He}^{2+}$ and $\mathrm{O}^{6+}$, emit at EUV and FUV wavelengths. Model calculations based on $\mathrm{O}^{q+}-\mathrm{H}_{2}$ interactions underline the important role of $\mathrm{O}^{6+}$ ions as line emitters (Kharchenko et al. 1998; Liu \& Schultz 1999). Both the FUSE and SOHO-UVCS observatories were used to search for the O VI doublet at $103 \mathrm{~nm}$ in five comets (Feldman 2005; Raymond et al. 2002; Weaver et al. 2002). The nondetection of what are predicted to be the brightest SWCX lines Kharchenko \& Dalgarno (2001) might be attributed to the relatively small field of view of FUSE, allowing observation of only a small fraction of the coma. As the four comets in these studies were all very active the O VI brightness might have very well peaked outside FUSE's field of view (Feldman 2005; Sasseen et al. 2006). The Cosmic Hot Interstellar Plasma Spectrometer (CHIPS) spectrograph was used to observe three comets between $9-26 \mathrm{~nm}$, with a peak resolution of $0.4 \mathrm{~nm}$ FWHM (Sasseen et al. 2006). Interestingly, the authors reported a $2 \sigma$ detection $\mathrm{O}$ VI in the spectrum of Comet C/2001 Q4 (NEAT). Experimental studies (Bodewits \& Hoekstra 2007; Miller et al. 2011) indicate that this O VI SWCX emission between $10-20 \mathrm{~nm}$ is another potent diagnostic of local solar wind velocities (Fig. 5).

Helium ion are approximately 200 times more abundant than the oxygen ions that drive cometary X-rays. Low resolution EUVE observations of several comets between $8-70$ $\mathrm{nm}$ allowed for the identification of O III-VI, C V, and He III SWCX emission lines Krasnopolsky \& Mumma 2001; Krasnopolsky et al. 2000). Helium charge exchange emission is dominated by two strong peaks; the He II (2p-1s) transition at $30.4 \mathrm{~nm}$ from one electron capture, and the He I $\left(1 \mathrm{~s} 2 \mathrm{p}-1 \mathrm{~s}^{2}\right)$ transition at $58.4 \mathrm{~nm}$ following two-electron capture (Bodewits et al. 2004, 2005). The ratio of these lines changes by more than three orders of magnitude for typical solar wind velocities between 100 to $1000 \mathrm{~km} \mathrm{~s}^{-1}$ (Fig. 5). This diagnostic was applied to EUVE observations of comets Hale-Bopp and Hyakutake to interpret SWCX emission in terms of local solar wind characteristics, providing an excellent window on what may be achieved once high resolution $\mathrm{X}$-ray spectroscopy becomes available.

\section{Conclusion}

When solar wind ions pass through an atmosphere they are neutralized via charge exchange reactions with the neutral gaseous species. These reactions depend strongly on target species and collision velocity. The resulting X-ray and UV emission is therefore a fingerprint of the underlying interaction, with many diagnostic qualities.

To fully explore those diagnostics one has to consider all aspects relevant for cometary X-ray emission: experimental studies of state selective charge exchange cross sections and the resulting line emission, observational techniques to use $\mathrm{X}$-ray telescopes that were certainly not designed to view objects within the solar system, and modeling of the prop- 
agation of the solar wind and its interaction with the gas in cometary comae. Together, these have greatly improved our understanding of the interaction of the solar wind with solar system objects and in more general, of physical processes in wind-environment collisions. The thorough understanding of cometary charge exchange emission has opened the door to the direct observation of more complex solar wind interactions such as those with Earth, Mars, Venus, Jupiter, and the heliosphere.

\section{References}

A'Hearn, M. F., Millis, R. L., Schleicher, D. G., Osip, D. J., \& Birch, P. V. 1995, Icarus (ISSN 0019-1035), 118, 223

Anderson, H., von Hellermann, M., Hoekstra, R., et al. 2000, Plasma Phys. Controll. Fusion., 40, 781

Beiersdorfer, P., Boyce, K. R., Brown, G. V., et al. 2003, Science, 300, 1558

Bingham, R., Dawson, J. M., Shapiro, V. D., Mendis, D. A., \& Kellet, B. J. 1997, Science, 275, 49

Bodewits, D., Christian, D. J., Torney, M., et al. 2007, Astronomy and Astrophysics, 469, 1183

Bodewits, D. \& Hoekstra, R. 2007, Physical Review A, 76, 6

Bodewits, D., Juhász, Z., Hoekstra, R., \& Tielens, A. G. G. M. 2004, ApJ. Lett., 606, L81

Bodewits, D., Tielens, A. G. G. M., Morgenstern, R., \& Hoekstra, R. 2005, NIMB, 235, 358

Brown, G., Beiersdorfer, P., Bodewits, D., et al. 2010, The Energetic Cosmos: From Suzaku to Astro-H Otaru, Japan June 29, 2009 through July 2, 2009

Bryans, P., Landi, E., \& Savin, D. W. 2009, ApJ , 691, 1540

Carter, J., Bodewits, D., Read, A. M., \& Immler, S. M. 2012, A\&A, in press

Carter, J., Sembay, S., \& Read, A. 2010, Monthly Notices of the Royal Astronomical Society, 402, 867

Carter, J. A. \& Sembay, S. 2008, Astronomy and Astrophysics Supplement Series (ISSN 0365-0138), 489, 837

Carter, J. A., Sembay, S., \& Read, A. M. 2011, Astronomy and Astrophysics, 527, 115

Christian, D. J., Bodewits, D., Lisse, C. M., et al. 2010, Astrophysical Journal Supplement Series, 187, 447

Cravens, T. E. 1997, Geophys. Res. Lett., 24, 105

-. 2002, Science, 296, 1042

Dennerl, K., Englhauser, J., \& Trümper, J. 1997, Science, 277, 1625

Dennerl, K. e. a. 2012, in prep

Ewing, I., Christian, D., Bodewits, D., et al. 2012, in prep.

Feldman, P. D. 2005, Physica Scripta (ISSN 0031-8949), 119,7

Fry, C. D., Dryer, M., Smith, Z., et al. 2003, J. Geophys. Res., 108, 5

Geiss, J., Gloeckler, G., von Steiger, R., et al. 1995, Science, 268, 1033

Haberli, R. M., Gombosi, T. I., DeZeeuw, D. L., Combi, M. R., \& Powell, K. G. 1997, Science, 276, 939
Hoekstra, R., Anderson, H., Bliek, F. W., et al. 1998, Plasma Phys. Controll. Fusion., 40, 1541

Isler, R. C. 1994, Plasma Physics and Controlled Fusion, 36, 171

Jorda, L., Crovisier, J., \& Green, D. W. E. 2008, Asteroids, 1405, 8046

Kharchenko, V. \& Dalgarno, A. 2000, J. Geophys. Res., 105,18351

—. 2001, ApJ. Lett., 554, L99

Kharchenko, V., Liu, W., \& Dalgarno, A. 1998, J. Geophys. Res., 103, 26687

Koutroumpa, D., Acero, F., Lallement, R., Ballet, J., \& Kharchenko, V. 2007, Astronomy and Astrophysics Supplement Series (ISSN 0365-0138), 475, 901

Koutroumpa, D., Lallement, R., Kharchenko, V., \& Dalgarno, A. 2008, arXiv, 0805, 3212

Krasnopolsky, V. 1997, Icarus, 128, 368

Krasnopolsky, V. A. 2004, Icarus, 167, 417

Krasnopolsky, V. A., Christian, D. J., Kharchenko, V., et al. 2002, Icarus, 160, 437

Krasnopolsky, V. A., Greenwood, J. B., \& Stancil, P. C. 2004, Space Science Reviews, 113, 271

Krasnopolsky, V. A. \& Mumma, M. J. 2001, ApJ, 549, 629

Krasnopolsky, V. A., Mumma, M. J., \& Abbott, M. J. 2000, Icarus (ISSN 0019-1035), 146, 152

Landi, E., Alexander, R. L., Gruesbeck, J. R., et al. 2012, ApJ , 744, 100

Lepri, S. T. \& Zurbuchen, T. H. 2004, J. Geophys. Res., 109, 1112

Lisse, C. M., Bodewits, D., Christian, D. J., et al. 2007, Icarus, in press

Lisse, C. M., Christian, D. J., Dennerl, K., et al. 2001, Science, 292, 1343

-. 2005, ApJ, 635, 1329

Lisse, C. M., Dennerl, K., Englhauser, J., et al. 1996, Science, 274, 205

Liu, W. \& Schultz, D. R. 1999, Ap.J., 526, 538

Miller, K. A., Smith, W. W., Ehrenreich, T., et al. 2011, The Astrophysical Journal, 742, 130

Mumma, M. J., Krasnopolsky, V. A., \& Abbott, M. J. 1997, ApJ. Lett., 491, L125

Neugebauer, M., Cravens, T. E., Lisse, C. M., et al. 2000, J. Geophys. Res., 105, 20949

Northrop, T. G., Lisse, C. M., Mumma, M. J., \& Desch, M. D. 1997, Icarus, 127, 246

Owens, A., Parmar, A. N., Oosterbroek, T., et al. 1998, ApJ. Lett., 493, L47

Raymond, J. C., Uzzo, M., Ko, Y.-K., et al. 2002, The Astrophysical Journal, 564, 1054

Sasseen, T. P., Hurwitz, M., Lisse, C. M., et al. 2006, ApJ , 650,461

Schultz, R., Owens, A., Rodríguez-Pascual, P. M., et al. 2006, Astronomy and Astrophysics Supplement Series (ISSN 0365-0138), 448, L53

Schulz, R., Stüwe, J. A., Tozzi, G. P., \& Owens, A. 2000, A\&A, 361, 359 
Schwadron, N. A. \& Cravens, T. E. 2000, ApJ, 544, 558

Uchida, M., Morikawa, M., Kubotani, H., \& Mouri, H. 1998, ApJ, 498, 863

von Hellermann, M., Mandl, W., Summers, H. P., et al. 1991, Plasma Phys. Controll. Fusion., 33, 18051

Weaver, H. A., Feldman, P. D., Combi, M. R., et al. 2002, ApJ. Lett., 576, L95

Wegmann, R. \& Dennerl, K. 2005, A\&A, 430, L33

Wegmann, R., Dennerl, K., \& Lisse, C. M. 2004, A\&A, 428, 647

Wegmann, R., Schmidt, H. U., Lisse, C. M., Dennerl, K., \& Englhauser, J. 1998, Planet. Space Sci., 46, 603

Wickramasinghe, N. C. \& Hoyle, F. 1996, Astroph. Space Sci., 239, 121

Willingale, R., O’Brien, P. T., Cowley, S. W. H., et al. 2006, ApJ, 649, 541

Wolk, S., Lisse, C. M., Bodewits, D., Christian, D. J., \& Dennerl, K. 2009, The Astrophysical Journal, 694, 1293

Zurbuchen, T. H., Fisk, L. A., Gloeckler, G., \& von Steiger, R. 2002, Geophys. Res. Lett., 29, 66 